Canadian

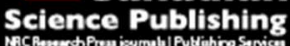

Applied Physiology, Nutrition, and Metabolism Physiologie appliquée, nutrition et métabolisme

\title{
Cortisol, blood pressure and heart rate responses to food intake were independent of physical fitness levels in women.
}

\begin{tabular}{|r|l|}
\hline Journal: & Applied Physiology, Nutrition, and Metabolism \\
\hline Manuscript ID & apnm-2015-0168.R2 \\
\hline Manuscript Type: & Article \\
\hline Date Submitted by the Author: & 15 -Jul-2015 \\
\hline Complete List of Authors: & $\begin{array}{l}\text { Jayasinghe, Sisitha; Deakin University, School of Exercise and Nutrition } \\
\text { Sciences } \\
\text { Torres, Susan; Deakin University, School of Exercise and Nutrition Sciences } \\
\text { Fraser, Steve; Deakin University, School of Exercise and Nutrition Sciences } \\
\text { Turner, Anne; Deakin University, School of Exercise and Nutrition Sciences }\end{array}$ \\
\hline Keyword: & physiology, nutrition, exercise, hormones, stress \\
\hline
\end{tabular}


1 Cortisol, blood pressure and heart rate responses to food intake were

2 independent of physical fitness levels in women.

$3 \quad$ 1Jayasinghe Sisitha U; sui@deakin.edu.au, ${ }^{1}$ Torres Susan J;

$4 \quad$ susan.torres@deakin.edu.au, ${ }^{1}$ Fraser Steve F; steve.fraser@deakin.edu.au, ${ }^{1} T u r n e r$

5 Anne I

6

7 Corresponding author

8 Dr Anne I Turner

9 Senior Lecturer

10 Centre for Physical Activity and Nutrition

11 School of Exercise and Nutrition Sciences

12 Deakin University, Melbourne, 3125, VIC, Australia

13 Phone: +61392446950

14 Email: anne.turner@deakin.edu.au

$16{ }^{1}$ Centre for Physical Activity and Nutrition, School of Exercise and Nutrition

17 Sciences, Deakin University, Burwood, VIC, Australia. 
21 Abstract

22 This research tested the hypothesis that women who had higher levels of physical fitness will have lower hypothalamo-pituitary adrenal (HPA) axis (cortisol) and sympatho-adrenal medullary (SAM) system (blood pressure and heart rate) responses to food intake compared with women who had low levels of physical fitness. Lower fitness $\left(\mathrm{n}=22 ; \mathrm{VO}_{2} \max =27.4 \pm 1.0 \mathrm{ml} / \mathrm{kg}^{*} \mathrm{~min}\right)$ and higher fitness $\left(\mathrm{n}=22 ; \mathrm{VO}_{2} \max =41.9 \pm 1.6 \mathrm{ml} / \mathrm{kg}^{*} \mathrm{~min}\right)$ women (aged $30-50$ years; in the follicular phase of the menstrual cycle) who participated in levels of physical activity which met $($ Lower fitness $=2.7 \pm 0.5 \mathrm{~h} /$ per week $)$ or considerably exceeded $($ Higher fitness $=$

Key words: HPA axis, SAM system, women, fitness, food intake, physical activity 
Food intake is a physiological challenge experienced by the human body several times per day. We and others have shown that food intake is a challenge that can activate both the sympatho-adrenal medullary (SAM) system (Chang et al. 2010; Cozzolino et al. 2010; Jayasinghe et al. 2014; Kawaguchi et al. 2002; Sauder et al.

Physical fitness status (Rimmele et al. 2009) and adiposity (Epel et al. 2000) are physiological conditions that can alter the activity of the stress pathways. Available evidence suggests that increased adiposity can be associated with higher HPA axis activity in response to food intake (Jayasinghe et al. 2014; Vicennati et al. 2002). Nevertheless, the influence of physical fitness status on physiological responses (both HPA axis and SAM system) to food intake has not been investigated before. Exercise brings about many health benefits including lowering progression to chronic disease by influencing heart rate, blood pressure and vascular endothelial functioning in response to stress (Hamer 2012; Throne et al. 2000; Tsatsoulis and Fountoulakis 2006). Moderating the HPA axis and SAM system responses to food 
69 intake may well be another avenue by which exercise exerts its protective capabilities against the development of chronic disease.

Activity of the SAM system increases cardiovascular (heart rate and blood pressure) activity (Grassi and Esler 1999). Activity of the HPA axis results in the secretion of cortisol from the adrenal cortex (Tilbrook 2007). Therefore, all of the parameters mentioned above can be used to measures the activity of the HPA axis and the SAM system. It is often best to include a collection of measures in order to fully characterise the activity of the stress pathways.

The aims of this study were to measure HPA axis and SAM system responses to food intake in women (in the follicular phase of the menstrual cycle) who differed in their levels of physical fitness. Given the marked influence of sex steroids on the activity of the stress pathways (Kajantie and Phillips 2006; Lustyk et al. 2010), this study was conducted in women only so as not to confound the results by including both genders. Since the change in levels of sex steroids during the menstrual cycle can also influence the activity of the stress pathways (Lustyk et al. 2010), this study investigated women in the same phase of the menstrual cycle (follicular phase) at the time of testing. It was hypothesised that women who had higher levels of physical fitness will have lower HPA axis and SAM system responses to food intake. 
90

91

92

\section{Materials and Methods}

Women $(n=44)$ aged 30-50 years were recruited using newspaper and online advertisements, emails, fliers in community centres and medical clinics. Exclusion criteria were prior diagnosis with Cushing's syndrome, any stress or anxiety disorder, depression, any diseases of the adrenal gland, type 2 diabetes, heart disease (including use of a pacemaker), high cholesterol, stroke or cancer. This information was self-reported by the participants via a telephone interview. Given the influence of sex steroids on activity of the stress pathways (Kajantie and Phillips 2006), postmenopausal women, peri-menopausal women and all women who were on any form of steroidal contraception (including oral contraceptives, steroidal implants and steroidal IUDs) were excluded from the study.

All participants provided written informed consent prior to participation in the study. All procedures were approved by the Human Research Ethics Committee of Deakin University (Project code: 2011-242) and conformed to the guidelines of the National Health and Medical Research Council's National Statement on Ethical Conduct in Human Research (2007).

\section{Experimental procedure}

Women reported to the laboratory on two separate days. The first visit was to obtain additional health information (details below), a fasting blood sample for the measurement of cardio-metabolic risk markers and to measure cardiorespiratory fitness (maximum oxygen consumption- $\mathrm{VO}_{2}$ max). The stress pathway activation in 
114 response to food intake (details below) was investigated on the second visit which occurred at least one week after the first visit.

\section{Day 1 testing}

118 Participants were given instructions to fast overnight (for at least 10 hours) prior to attending the laboratory. Day 1 testing was completed between 0600h $-1200 \mathrm{~h}$. Weight was recorded in kilograms to the nearest $0.1 \mathrm{~kg}$ with digital scales (TANITA, Wedderburn, Melbourne, Australia) on a firm surface. Height was measured to the nearest millimetre using a freestanding stadiometer (Measurement Concepts, North Bend, Australia). Participants were not wearing shoes in both measurements. BMI was calculated as weight $(\mathrm{kg})$ divided by height $(\mathrm{m})$ squared. Women whose BMI fell outside the range $18-30\left(\mathrm{~kg} / \mathrm{m}^{2}\right)$ were excluded from the study. Resting blood pressure was measured four times (Criticare systems Inc, Wisconsin, USA) at 2 min intervals and the average of the last three measurements were used to confirm whether resting blood pressure was within the required range $(<160 \mathrm{mmHg}$ for systolic and $<90 \mathrm{mmHg}$ for diastolic). This threshold for systolic was used since isolated systolic blood pressure of $>160 \mathrm{mmHg}$ is considered by the Heart Foundation of Australia as the point at which anti-hypertensive medication should be recommended (Heart Foundation 2008). Hypertension is defined in the Australian Heart Foundation Guide to Management of Hypertension as $>140 / 90 \mathrm{mmHg}$. None 134 of the women recruited for this study exceeded a resting blood pressure of 135 140/90mmHg. 
137 All eligible participants were subsequently subjected to a single venipuncture in a

138 vein of the antecubital fossa of the forearm using a sterile vacuette safety blood

139 collection set (GreinerBio-One $\mathrm{GmbH}$, Kremsmunster, Austria). Blood was collected

140 into a 9ml serum separator tube (GreinerBio-One $\mathrm{GmbH}$, Kremsmunster, Austria)

141 and two 2ml plasma EDTA (GreinerBio-One $\mathrm{GmbH}$, Kremsmunster, Austria) tubes.

142 Serum was sent to a commercial pathology laboratory (Dorevitch, Melbourne,

143 Australia) for analysis of lipid profile (total cholesterol, high density lipoprotein, low

144 density lipoprotein and triglycerides), fasting serum glucose, and C-reactive protein.

Participants were allowed to have a snack (a selection of foods from muesli bars, nuts, dried fruit and juice boxes were made available) after collection of blood. A

148 Physical Activity Readiness Questionnaire (PAR-Q) was filled in at this time to assess if it was safe for each participant to undertake a $\mathrm{VO}_{2}$ max test. Participants also filled out an International Physical Activity Questionnaire (IPAQ) (Bauman et al. 2009) to measure levels of high and moderate intensity physical activity, a StateTrait Anxiety Inventory (STAI) (Spielberger et al. 1983) to measure levels of anxiety and a Beck Depression Inventory (BDI-ii) (Beck et al. 2006) to measure levels of depressive symptoms. Water was available ad libitum to all participants throughout

155 the testing session. This was immediately followed by the graded $\mathrm{VO}_{2}$ max test on an electronically braked cycle ergometer (Lode N.V. Groningen, Netherlands). After ranking women by $\mathrm{VO}_{2}$ max score, a median split was then used to allocate women evenly into a higher fitness group $(n=22)$ and a lower fitness group $(n=22)$. 
161

162

163

164

\section{5}

166

167

168

\section{Day 2 testing}

Participants were in their mid-follicular phase of the menstrual cycle at the time of this testing session. Mid follicular phase was defined as Days 5-9 of the menstrual cycle, inclusive, where Day 1 was the first day of menses onset (Lustyk et al. 2010). Participants were asked to abstain from smoking, ingesting any caffeine containing beverages (e.g. tea, coffee, cola), liquorice, alcohol or drugs (except for any regular medications) and from strenuous physical activity during the 12 hours prior to Day 2 testing.

Participants were instructed to arrive at the laboratory at 1100h. Between 1100h1145h, measurements of waist and hip circumference and body fat (TANITA, Wedderburn, Melbourne, Australia) were obtained and participants were asked to fill in a background questionnaire about their alcohol consumption and physical activity in the week preceding the testing day. Waist circumference was measured at the midpoint between the last rib and the anterior superior iliac spine using a tape measure and hip circumference was measured at the widest point of the gluteal area (Dettwyler 1993). Waist to hip ratio was calculated by dividing waist circumference by hip circumference. Also during this period, an intra-venous catheter (Smiths Medical, Ohio, USA) was inserted into an antecubital vein of the forearm for subsequent sampling of blood.

Participants were given a test meal (details below) at $1200 \mathrm{~h}$. They were allowed to consume food between 1200h-1230h. Blood samples were collected every 15 min between 1145h-1400h. Systolic blood pressure (SBP), diastolic blood pressure (DBP), mean arterial pressure (MAP) and heart rate (HR) were also measured at 15 
185 min intervals during this period using a clinical blood pressure monitor (Criticare 186 systems Inc, Wisconsin, USA) to gauge the activity levels of the SAM system.

187 Although not considered as direct measures, SBP, DBP, MAP and HR have been used as proxy measures of SAM system activity under different circumstances (de

Geus et al. 1993; Grassi and Esler 1999; Webb et al. 2013). We have also

previously shown that food intake can cause significant changes in heart rate and

blood pressure (Jayasinghe et al. 2014). Participants were allowed a break to use sampling.

\section{Test meal}

Lithium Heparin tubes (GreinerBio-One $\mathrm{GmbH}$, Kremsmunster, Austria) were used to collect blood samples $(5 \mathrm{ml})$ for cortisol assays. All tubes were spun at $3000 \mathrm{rpm}$ for $6 \mathrm{~min}$. Plasma was separated and stored at $-80^{\circ} \mathrm{C}$ until assay.

The test meal consisted of lunch made by the participants from a choice of standardised ingredients including bread, margarine, processed meat (ham or chicken), tomato, cucumber, cheese, nuts, fruit bars and a fruit drink (juice box). Water was available ad libitum. The investigator took records of the foods consumed. Dietary intake was determined using household measures. Total energy, macronutrient and sodium intake was determined using FoodWorks professional edition (version 7; Xyris software, Brisbane, Queensland, Australia). 
Plasma cortisol assays

211 Plasma concentrations of cortisol were measured using a radio immunoassay

212 (Demeditec Diagnostics, Kiel, Germany). Forty-four assays were conducted. The

213 intra-assay coefficient of variation was $9.8 \%$ at $92 \mathrm{ng} / \mathrm{mL}$ and $9.4 \%$ at $193 \mathrm{ng} / \mathrm{ml}$.

214 The inter-assay coefficient of variation was $10.7 \%$ at $146 \mathrm{ng} / \mathrm{ml}$ and $10.2 \%$ at 137 $215 \mathrm{ng} / \mathrm{ml}$.

\section{Statistical analysis}

\section{Preliminary analysis}

219 Pre- treatment salivary cortisol was defined as the concentration of cortisol in the 220 sample collected at $1200 \mathrm{~h}$. Pre- treatment SBP, DBP, MAP and HR were defined as 221 the average of values recorded at $1145 \mathrm{~h}$ and $1200 \mathrm{~h}$. Peak height for cortisol was defined as the highest value obtained for each individual between $1215 \mathrm{~h}-1400 \mathrm{~h}$, inclusive. Peak height for all cardiovascular parameters was defined as the highest value obtained between 1215h-1330h. Data from 1345h- 1400h were not used in this calculation because of the apparent effects on cardiovascular parameters of physical movements during the bathroom break. Reactivity was calculated by subtracting the pre-treatment value from the peak height for all parameters. Area under the curve (with respect to increase) was calculated for cortisol using all values between $1200 \mathrm{~h}-1400 \mathrm{~h}$ and for SBP, DBP, MAP and HR using values between $1200 \mathrm{~h}-1330 \mathrm{~h}$ after the subtraction of the pre-treatment value from each

231 data point. Area under the curve for all parameters was calculated using the 
232 trapezoid rule utilising Sigmaplot 12.5 graphing software (Systat Software Inc., 233 California, USA).

\section{Analysis}

236 Data were analysed using the Statistical Package for the Social Sciences software 237 version 21.0 for Windows (SPSS. Inc, Chicago, USA). Kolmogorov-Smirnov and 238 Shapiro -Wilk tests were conducted to test for normality. Tests for homogeneity of 239 variance were conducted using Levene's test of equality of error variances.

240 Descriptive characteristics were compared between groups using univariate analysis

241 of variance. Plasma cortisol, blood pressure and heart rate were compared within 242 and between subjects using repeated measures analysis of variance. The within 243 subjects factor was time and the between subjects factor was treatment. Derived 244 plasma cortisol and cardiovascular parameters (pre- treatment, peak height, reactivity and area under the curve) were compared between groups using univariate analysis of variance. $\mathrm{P}<0.05$ was considered statistically significant.

We estimated that 32 participants in total were needed to find a difference between groups in salivary cortisol of the same magnitude as that found by Klaperski and colleagues (Klaperski et al. 2013) with a significance level of 0.05 and a power of $25190 \%$. 


\section{Results}

255

256

257

258

259

260

261

262

263

264

265

266

267

268

269

270

271

272

273

274

275

276

277

\section{Participants}

A total of 44 women completed the study. Women were ranked according to their $\mathrm{VO}_{2}$ max score and a median split was used to allocate women to two even groups (lower fitness group; $n=22$ and higher fitness group; $n=22$ ). One woman in the higher fitness group had to be excluded from the cortisol analyses due to a blocked cannula which prevented the collection of several blood samples.

\section{Participant characteristics}

The labels given to the groups in this study were relative terms used to differentiate between the higher $\mathrm{VO}_{2}$ max group and the lower $\mathrm{VO}_{2}$ max group. According to the American Heart Association cardiorespiratory fitness classification criteria (AHA 1972), women in our lower fitness group would be classified as "fair" to "average" whereas women in our higher fitness group would be classified as "good" to "high". Women in the higher fitness group had significantly higher $\mathrm{VO}_{2}$ max levels and participated in a significantly higher number of hours of moderate and vigorous intensity physical activity compared with the women in the lower fitness group $(p<0.01$ for both; Table 1$)$. The number of hours of moderate and vigorous intensity physical activity undertaken by lower fitness women $(2.7 \pm 0.5 \mathrm{~h} /$ per week $)$ was sufficient to meet the physical activity recommendations of the American Heart Association (AHA) and the number of hours undertaken by higher fitness women (7.1 $\pm 1.3 \mathrm{~h} /$ per week) considerably exceeded these recommendations (Centers for Disease Control and Prevention 2008). Percentage body fat and waist 
278 circumference were significantly lower in the higher fitness group compared with the 279 lower fitness group ( $p<0.05$ for both). Furthermore, the lower fitness group had significantly more abdominal body fat compared with the higher fitness group as indicated by the waist to hip ratio ( $p=0.004 ;$ Table 1$)$. Higher fitness women had significantly lower serum triglyceride levels, serum $\mathrm{CHOL/HDL}$ ratio, serum glucose concentrations and HOMA-IR compared with lower fitness women $(p<0.05$ for all; scores (Table 2).

Test meal

Lower fitness and higher fitness women consumed similar amounts of total energy, protein, carbohydrate, fat and sodium (Table 3). There were no significant carbohydrates and $19 \%$ fat.

\section{Plasma cortisol}

Plasma concentrations of cortisol in lower fitness and higher fitness women are shown in Figure 1 and Table 4. Repeated measures analysis of variance revealed that there was a significant effect of time $(F(9,33)=2.657, p=0.05$; Figure 1). 
concentrations was significantly higher $(p<0.001)$ than the pre-treatment $(137.4 \pm$ $10.4 \mathrm{ng} / \mathrm{mL}$ ) cortisol concentrations. This represented a $27 \%$ increase from the pretreatment cortisol concentrations.

In response to lunch, plasma concentrations of cortisol did not differ significantly between lower fitness and higher fitness women (time*treatment, $F(9,33)=0.488, p$ $=0.882 ;$ Figure 1). Furthermore, there was no significant differences between groups in peak height, cortisol reactivity and area under the curve ( $p>0.05$ for all; Table 4). There was also no significant between subjects effect indicating that overall, higher fitness women had similar cortisol levels compared with lower fitness women $(F(9,33)=0.042, p=0.839)$.

\section{Cardiovascular parameters}

Cardiovascular parameters in lower fitness and higher fitness women are presented in Figure 2 and Table 5.

\section{Systolic blood pressure}

There was a significant effect of time $(F(9,34)=5.450, p<0.001)$ for systolic blood pressure (Figure 2a). Overall (both groups combined), the peak height of systolic blood pressure $(120 \pm 3 \mathrm{mmHg})$ was significantly higher than the pre-treatment systolic blood pressure $(108 \pm 2 \mathrm{mmHg})(p<0.001)$. This represents a $12 \%$ increase.

Systolic blood pressure in response to the lunch did not differ significantly between lower fitness and higher fitness women (time ${ }^{*}$ treatment, $F(9,34)=0.961, p=0.472$; 
325 Figure 2a). This lack of difference of the response between groups was further

326 illustrated by there being no difference in peak height, reactivity and area under the

327 response curve for systolic blood pressure between the two groups ( $p>0.05$ for

328 both; Table 5). There was also no significant between subjects effect indicating that

overall, higher fitness women had similar systolic blood pressure compared with the lower fitness women (treatment effect, $F(9,34)=3.627, p=0.064)$.

\section{Diastolic blood pressure}

333

There was a significant effect of time $(F(9,34)=7.915, p<0.001)$ and no treatment effect $(p=0.180)$ for diastolic blood pressure (Figure $2 b)$. Overall (both groups combined), the peak height of diastolic blood pressure $(72 \pm 2 \mathrm{mmHg}$ ) was significantly higher than the pre-treatment diastolic blood pressure $(63 \pm 1 \mathrm{mmHg})$ $(p<0.001)$. This represents a $14 \%$ increase.

Diastolic blood pressure in response to the lunch did not differ significantly between lower fitness and higher fitness women (time treatment, $F(9,34)=0.514, p=0.864$; Figure $2 \mathrm{~b}$ ). Furthermore, diastolic blood pressure peak height, reactivity and area under the curve did not differ between the two groups (Table 5). There was also no significant between subjects effect indicating that overall, higher fitness women had similar diastolic blood pressure compared with the lower fitness women (treatment effect, $F(9,34)=1.862, p=0.180)$.

\section{Mean Arterial pressure}


349 There was a significant effect of time $(F(9,34)=7.657, p<0.001)$ for mean arterial

350 pressure (Figure 2c). Overall (both groups combined), the peak height of mean

351 arterial pressure $(88 \pm 2 \mathrm{mmHg})$ was significantly higher than the pre-treatment mean

352 arterial pressure $(80 \pm 2 \mathrm{mmHg})(p<0.001)$. This represents an $11 \%$ increase.

Mean arterial pressure in response to the lunch did not differ significantly between lower fitness and higher fitness women (time*treatment, $F(9,34)=0.590, p=0.805$;

356 Figure 2c). Peak height, reactivity and area under the response curve for mean arterial pressure were similar between the groups ( $p>0.05$ for both; Table 5$)$. There was also no significant between subjects effect indicating that overall, higher fitness women had similar mean arterial pressures compared with the lower fitness group ( $F$ $(9,34)=3.205, p=0.081)$

\section{Heart rate}

There was a significant effect of time $(F(9,34)=4.933, p<0.001)$ for heart rate

365 (Figure 2d). Overall (both groups combined), the peak height of heart rate (75 \pm 2

$\mathrm{mmHg})$ was significantly higher from the pre-treatment heart rate $(66 \pm 2 \mathrm{mmHg})$

$367(p<0.001)$. This represents a $14 \%$ increase.

368

Heart rate in response to the lunch did not differ significantly between lower fitness and higher fitness women (time*treatment, $F(9,34)=1.319, p=0.225$; Figure $2 d$ ).

371 Nevertheless, higher fitness women had a significantly lower $(p=0.005)$ peak height of the heart rate response compared with lower fitness women. Nevertheless, heart 
373 rate reactivity was not different $(p=0.084)$ between higher fitness and lower fitness

374 women. Pre-treatment values and area under the curve did not differ between the

375 two groups (Table 5). Overall, lower fitness women had significantly higher levels of

376 heart rate compared with the higher fitness women as indicated by the significant

377 treatment effect $(F(9,34)=7.703, p=0.008)$.

378 


\section{Discussion}

380 Our hypothesis that women who had higher levels of physical fitness would have

381

382 lower SAM system and HPA axis responses to the ingestion of a standardised lunch compared with women who had lower levels of fitness was not supported. While all of the parameters tested (plasma cortisol, blood pressure and heart rate) increased significantly in response to food intake, none of these responses differed between the groups. These results suggest that there is comparable SAM system and HPA axis activity in response to food intake in women with different physical fitness statuses. Indeed, it seems that HPA axis and SAM system responses to food intake are independent of physical fitness status in women.

In the present study, there was a substantial elevation of cortisol in response to lunch as indicated by the time effect and the significant increase in cortisol levels from baseline to the peak of the response (27\%; both groups combined), despite there being no difference between lower fitness and higher fitness groups in this response. In an earlier experiment (Jayasinghe et al. 2014) we observed a significant HPA axis response (salivary cortisol) to food intake in overweight/obese men but no response in lean men. While the percentage increase of cortisol in overweight/obese men ( $86 \%$ ) was substantially higher than the percentage increase of cortisol in women (27\%; both groups combined), the men's study measured salivary cortisol whereas the current study measured plasma cortisol. Since salivary cortisol indicates the free fraction of cortisol (a small proportion of total cortisol), it is possible the differences in percentage increases that were observed may have been due to the differences in HPA axis activity measures (saliva vs plasma) that were 
403

404

405

406

407

408

409

410

411

412

413

414

415

416

417

418

419

420

421

422

423

424

425

426

427

used. As such, it is not meaningful to make direct comparisons of the percentage increases between the studies.

In the current experiment, all SAM system parameters in both groups were elevated in response to lunch. These increases are in accordance with the reports of Harthoorn et al who found increases in sympathetic nervous system (heart rate and salivary alpha amylase) activity after ingestion of a standardised meal (15-20\% protein, $35-40 \%$ fat and $40-45 \%$ carbohydrate) in a group of healthy men and women (Harthoorn \& DransWeld, 2008). There is also evidence suggesting that ingestion of food can cause significant changes in parameters of heart rate variability (high frequency power, low frequency power and low to high frequency ratio) (Kawaguchi et al. 2002). Previous reports also indicated that the rise in sympathetic activity following a meal is dependent on the nutrient content of the foods ingested. For instance, Tentolouris et al (2003) reported increases in sympathetic activity (measured via plasma noradrenaline and heart rate variability) in lean healthy young women, only after consuming a high (95\%) carbohydrate meal (Tentolouris et al. 2003). Nevertheless, these elevations are indicative of the physiological demands that food intake places on the sympathetic nervous system activity (Jager et al., 1986). There was a reduction in systolic blood pressure, diastolic blood pressure and mean arterial pressure in the postprandial period in both groups (i.e., 1230h$1330 \mathrm{~h}$ in the current experiment) which may indicate a reduction in resistance to blood flow in the mesenteric vessels and perhaps even an indication that the satiety hormones are having an inhibitory effect on the sympathetic nervous system during this period (Burcelin, 2005; Fan et al., 2004). It has also been suggested that down regulation of catecholamines (in particular noradrenaline) could be a possible 
428 mechanism of this postprandial hypotensive effect (Kawaguchi et al. 2002).

429 Nevertheless, the absence of any significant differences in SAM system parameters

430 between higher fitness and lower fitness women in the current experiment suggests

431 that, on the whole, the post prandial sympathetic activity is independent of physical

432 fitness statuses in women.

434 Abdominal obesity can have a significant impact on the activity of the stress

435 pathways (Epel et al., 2000; Katz et al., 2000). Vicennati and colleagues reported

436 that high carbohydrate meals ( $89 \%$ carbohydrate, $11 \%$ protein, $0 \%$ fat) can result in

437 a significant HPA axis response in women who predominantly had a visceral body fat

438 distribution compared with women with peripheral body fat distribution and normal

439 weight healthy controls (Vicennati et al., 2002). Despite there being no difference

440 between the groups in BMI in the current study, waist to hip ratios and percent body

441 fat levels indicate that the lower fitness women had significantly more abdominally

442 based body fat compared with their fitter counterparts. However, it should be noted

443 that the WHR of women who had visceral body fat in Vicennati and colleagues

444 research, was much higher $(0.92 \pm 0.01)$ than the WHR of lower fitness women

$445(0.84 \pm 0.01)$ in the current experiment. Furthermore, it appears that obesity does not

446 influence food intake related SAM system activity in the same way as it does

447 influence food intake evoked HPA axis responses. For instance, Tentolouris and

448 colleagues reported no increases in sympathetic activity (measured via plasma

449 noradrenaline and heart rate variability) in obese young women after the

450 consumption of a high (95\%) carbohydrate meal (Tentolouris et al. 2003).

451 Nevertheless, given that there were no significant differences between the groups in

452 SAM and HPA axis activity in the current study, it suggests that having greater 
453 quantities of abdominally based body fat may not accentuate SAM system and HPA axis responses to food intake in lower fitness women.

None of the previous experiments in the area had investigated the effects of physical fitness status (objectively measured via maximal oxygen consumption) on SAM system and HPA axis response to food intake. Higher fitness women in the current study had an average $\mathrm{VO}_{2}$ max level of $41.9 \pm 1.6 \mathrm{ml} / \mathrm{kg}^{*} \mathrm{~min}$. Therefore, it is possible that women with even higher maximal oxygen consumption levels would be required to observe a different response pattern to food intake. This could be studied in percentage of daily energy expenditure or resting metabolic rate.

475 This experiment showed that physical fitness status (objectively measured via maximal oxygen consumption) in women (in the follicular phase of the menstrual 
477 cycle) did not have a significant influence on HPA axis and SAM system activity after 478 the ingestion of a meal consisting of $20 \%$ protein, $61 \%$ carbohydrates and $19 \%$ fat. 479 This suggests that ingesting a standardised meal does not result in excessive HPA 480 axis and SAM system activation in women of 30-50 years who have lower levels of 481 physical fitness compared with age matched women who have higher levels of 482 physical fitness.

483

\section{Acknowledgements}

485 Authors thank Mais Hussein for technical support throughout the experiment and all 486 the subjects for participating in this research. 
489

490

491

492

493

494

495

496

497

498

499

500

501

502

503

504

505

506

507

508

509

510

511

512

513

514

515

516

517

518

519

520

521

522

523

524

525

526

527

528

529

530

531

\section{References}

AHA 1972, Exercise testing and training of apparently healthy individuals: a handbook for physicians, American Heart Assoc.

Bauman, A., Ainsworth, B.E., Bull, F., Craig, C.L., Hagstromer, M., Sallis, J.F., et al. 2009. 'Progress and pitfalls in the use of the International Physical Activity Questionnaire (IPAQ) for adult physical activity surveillance'. Journal of physical activity \& health. 6(1): S5.

Beck, A.T., Steer, R.A. and Brown, G.K. 2006, Beck Depression Inventory Manual, Swedish version, Sandviken: Psykologiförlaget, Stockholm.

Carroll, D., Phillips, A.C. and Der, G. 2008. 'Body mass index, abdominal adiposity, obesity, and cardiovascular reactions to psychological stress in a large community sample'. Psychosom. Med. 70(6): 653-660.

Centers for Disease Control and Prevention 2008, Centers for Disease Control and Prevention, Physical Activity Guidelines for Americans, <http://www.cdc.gov/physicalactivity/everyone/guidelines/adults.html>.

Chang, C., Ko, C., Lien, H. and Chou, M. 2010. 'Varying postprandial abdominovagal and cardiovagal activity in normal subjects'. Neurogastroenterol. Motil. 22(5): 546-e119.

Chida, Y. and Hamer, M. 2008. 'Chronic psychosocial factors and acute physiological responses to laboratory-induced stress in healthy populations: a quantitative review of 30 years of investigations'. Psychol. Bull. 134(6): 829.

Cozzolino, D., Furlan, R., Gruosso, D., Di Maggio, C., Miraglia del Giudice, E., Torella, R., et al. 2010. 'Effects of a mixed meal on hemodynamics and autonomic control of the heart in patients with type 1 diabetes'. J. Clin. Endocrinol. Metab. 95(1): 194-200.

de Geus, E.J., Van Doornen, L.J. and Orlebeke, J.F. 1993. 'Regular exercise and aerobic fitness in relation to psychological make-up and physiological stress reactivity'. Psychosom. Med. 55(4): 347363.

Dettwyler, K., Lohman,TG, Roche,AF, Martorell,R 1993. 'Anthropometric Standardization Reference Manual, Abridged Edition '. Am. J. Phys. Anthropol. 92(2): 239-241.

Epel, E.S., McEwen, B., Seeman, T., Matthews, K., Castellazzo, G., Brownell, K.D., et al. 2000. 'Stress and body shape: Stress-induced cortisol secretion is consistently greater among women with central fat'. Psychosom. Med. 62(5): 623-632.

Gibson, E.L., Checkley, S., Papadopoulos, A., Poon, L., Daley, S. and Wardle, J. 1999. 'Increased salivary cortisol reliably induced by a protein-rich midday meal'. Psychosom. Med. 61(2): 214-224. 
Grassi, G. and Esler, M. 1999. 'How to assess sympathetic activity in humans'. J. Hypertens. 17(6): 719-734.

Hamer, M. 2012. 'Psychosocial Stress and Cardiovascular Disease Risk: The Role of Physical Activity'. Psychosom. Med. 74(9): 896-903.

Hamer, M. and Steptoe, A. 2011. 'Cortisol responses to mental stress and incident hypertension in healthy men and women'. J. Clin. Endocrinol. Metab. 97(1): E29-E34.

Heart Foundation 2008, Guide to management of hypertension, Heart Foundation Australia.

Jayasinghe, S.U., Torres, S.J., Nowson, C.A., Tilbrook, A.J. and Turner, A.I. 2014. 'Cortisol, alpha amylase, blood pressure and heart rate responses to food intake in men aged 50-70 years: importance of adiposity'. BMC Obesity. 1(1): 14.

Kajantie, E. and Phillips, D.I.W. 2006. 'The effects of sex and hormonal status on the physiological response to acute psychosocial stress'. Psychoneuroendocrinology. 31(2): 151-178.

Kawaguchi, R., Nomura, M., Miyajima, H., Nakaya, Y., Mouri, S. and Ito, S. 2002. 'Postprandial hypotension in elderly subjects: spectral analysis of heart rate variability and electrogastrograms'. J. Gastroenterol. 37(2): 87-93.

Klaperski, S., von Dawans, B., Heinrichs, M. and Fuchs, R. 2013. 'Does the level of physical exercise affect physiological and psychological responses to psychosocial stress in women?'. Psychol. Sport Exerc. 14(2): 266-274.

Lustyk, M.K.B., Olson, K.C., Gerrish, W.G., Holder, A. and Widman, L. 2010. 'Psychophysiological and neuroendocrine responses to laboratory stressors in women: Implications of menstrual cycle phase and stressor type'. Biol. Psychol. 83(2): 84-92.

Martens, M.J.I., Rutters, F., Lemmens, S.G.T., Born, J.M. and Westerterp-Plantenga, M.S. 2010. 'Effects of single macronutrients on serum cortisol concentrations in normal weight men'. Physiol. Behav. 101(5): 563-567.

Rimmele, U., Seiler, R., Marti, B., Wirtz, P.H., Ehlert, U. and Heinrichs, M. 2009. 'The level of physical activity affects adrenal and cardiovascular reactivity to psychosocial stress'. Psychoneuroendocrinology. 34(2): 190-198.

Sauder, K.A., Johnston, E.R., Skulas-Ray, A.C., Campbell, T.S. and West, S.G. 2012. 'Effect of meal content on heart rate variability and cardiovascular reactivity to mental stress'. Psychophysiology. 49(4): 470-477. 
575 Spielberger, C., Gorsuch, R., Lushene, R., Vagg, P. and Jacobs, G. 1983, Manual for the State-Trait 576 Anxiety Inventory. 1983 Palo Alto, CA: Consulting Psychology Press.

577
Tentolouris, N., Tsigos, C., Perea, D., Koukou, E., Kyriaki, D., Kitsou, E., et al. 2003. 'Differential effects of high-fat and high-carbohydrate isoenergetic meals on cardiac autonomic nervous system activity in lean and obese women'. Metabolism. 52(11): 1426-1432.

Throne, L.C., Bartholomew, J.B., Craig, J. and Farrar, R.P. 2000. 'Stress reactivity in fire fighters: An exercise intervention'. Int. J. Stress Manage. 7(4): 235-246.

Tilbrook, A.J. 2007, Encylopedia of stress, second edition edn, vol. 2, Neuropeptides, stress related.

Tsatsoulis, A. and Fountoulakis, S. 2006, 'The protective role of exercise on stress system dysregulation and comorbidities', in GP Chrousos \& C Tsigos (eds), Stress, Obesity, and Metabolic Syndrome, vol. 1083, pp. 196-213.

Vicennati, V., Ceroni, L., Gagliardi, L., Gambineri, A. and Pasquali, R. 2002. 'Response of the hypothalamic-pituitary-adrenocortical axis to high-protein/fat and high-carbohydrate meals in women with different obesity phenotypes'. J. Clin. Endocrinol. Metab. 87(8): 3984-3988.

Webb, H.E., Rosalky, D.S., Tangsilsat, S.E., McLeod, K.A., Acevedo, E.O. and Wax, B. 2013. 'Aerobic fitness affects cortisol responses to concurrent challenges'. Med. Sci. Sports Exerc. 45(2): 379-386. 
599 Tables

600 Table 1: Mean ( \pm SEM) descriptive characteristics of lower and higher fitness women

\begin{tabular}{|c|c|c|c|}
\hline & $\begin{array}{l}\text { Lower fitness } \\
(n=22)\end{array}$ & $\begin{array}{l}\text { Higher fitness } \\
(n=22)\end{array}$ & $p$ value ${ }^{*}$ \\
\hline Age (years) & $40.4 \pm 1.4$ & $38.1 \pm 1.3$ & 0.233 \\
\hline $\mathrm{VO}_{2} \max \left(\mathrm{ml} / \mathrm{kg}^{*} \min \right)$ & $27.4 \pm 1.0$ & $41.9 \pm 1.6$ & $<0.001$ \\
\hline Physical activity (hours) & $2.7 \pm 0.5$ & $7.1 \pm 1.3$ & 0.004 \\
\hline Weight (kg) & $62.6 \pm 2.3$ & $62.2 \pm 1.6$ & 0.833 \\
\hline BMI $\left(\mathrm{kg} / \mathrm{m}^{2}\right)$ & $23.1 \pm 0.7$ & $22.2 \pm 0.4$ & 0.241 \\
\hline$\%$ Fat & $30.3 \pm 1.4$ & $25.5 \pm 1.2$ & 0.013 \\
\hline Waist circumference $(\mathrm{cm})$ & $82.5 \pm 2.2$ & $76.4 \pm 1.3$ & 0.021 \\
\hline Hip circumference (cm) & $97.5 \pm 1.6$ & $96.1 \pm 1.2$ & 0.481 \\
\hline WHR & $0.84 \pm 0.01$ & $0.80 \pm 0.01$ & 0.004 \\
\hline Resting HR (bpm) & $71 \pm 2$ & $66 \pm 2$ & 0.089 \\
\hline Resting SBP (mmHg) & $115 \pm 3$ & $114 \pm 2$ & 0.926 \\
\hline Resting DBP (mmHg) & $70 \pm 2$ & $67 \pm 2$ & 0.232 \\
\hline \multicolumn{4}{|c|}{ * univariate analysis of variance } \\
\hline
\end{tabular}


603 Table 2: Mean $( \pm S E M)$ of cardio-metabolic risk markers and mental health scores in 604 lower fitness and higher fitness women

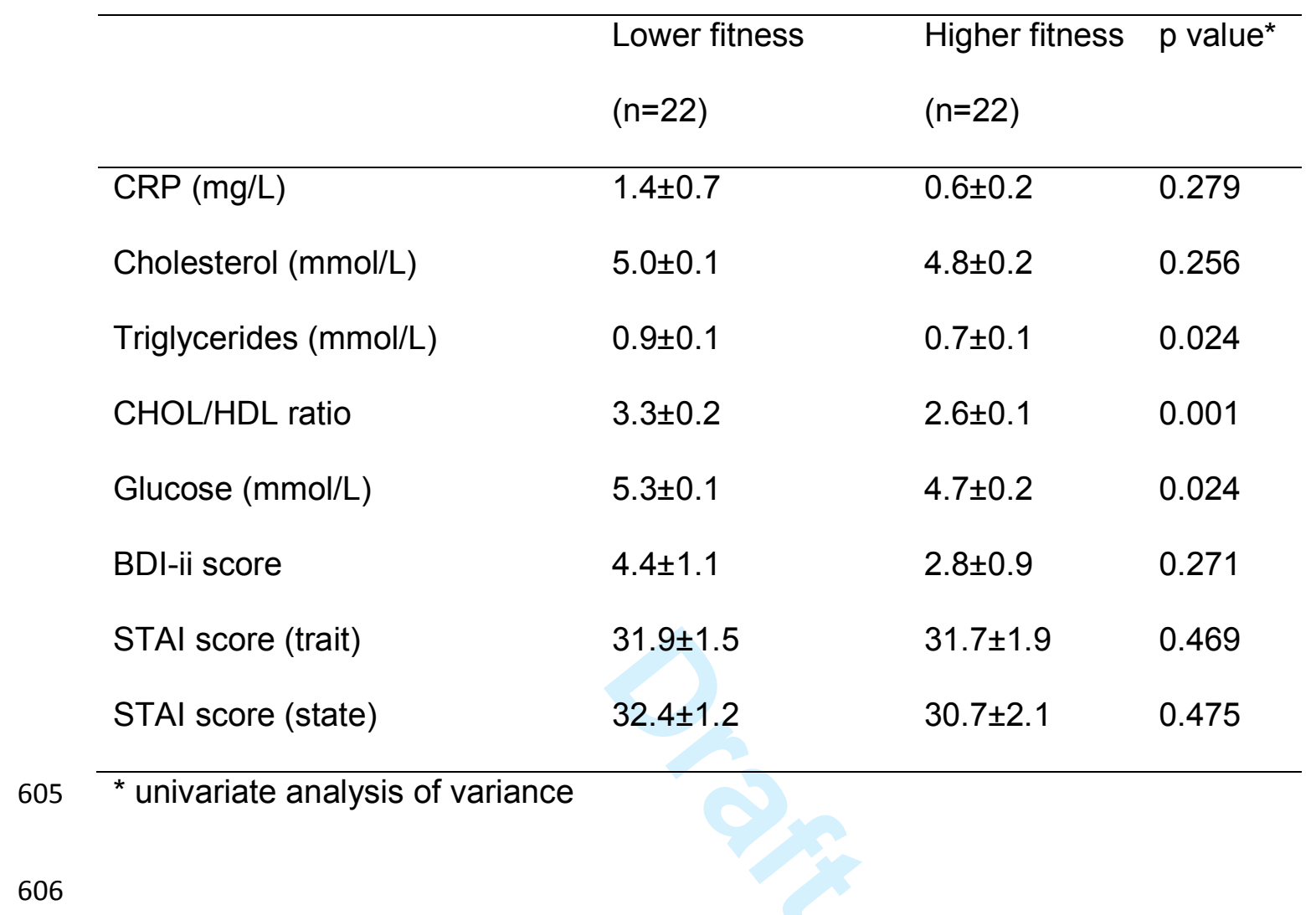


607 Table 3: Mean ( \pm SEM) total energy, macronutrient and sodium intake in lower 608 fitness and higher fitness women.

\begin{tabular}{llll}
\hline & $\begin{array}{l}\text { Lower fitness } \\
(\mathrm{n}=22)\end{array}$ & $\begin{array}{l}\text { Higher fitness } \\
(\mathrm{n}=22)\end{array}$ & p value* \\
\hline Total energy $(\mathrm{kJ})$ & $2047 \pm 162$ & $2094 \pm 116$ & 0.811 \\
Protein $(\mathrm{g})$ & $19.7 \pm 1.8$ & $19.3 \pm 1.6$ & 0.847 \\
Carbohydrate $(\mathrm{g})$ & $58.9 \pm 4.6$ & $59.6 \pm 3.3$ & 0.901 \\
Fat $(\mathrm{g})$ & $17.5 \pm 2.3$ & $19.2 \pm 2.0$ & 0.592 \\
Sodium $(\mathrm{mg})$ & $1047 \pm 115$ & $860 \pm 72$ & 0.176 \\
\hline
\end{tabular}


611 Table 4. Mean $( \pm S E M)$ pre-treatment cortisol, peak height of cortisol, cortisol

612 reactivity and area under the curve for lower fitness and higher fitness women

\begin{tabular}{llll}
\hline & $\begin{array}{l}\text { Lower fitness } \\
(\mathrm{n}=22)\end{array}$ & $\begin{array}{l}\text { Higher fitness } \\
(\mathrm{n}=21)\end{array}$ & $\mathrm{p}$ value $^{*}$ \\
\hline Pre-treatment $(\mathrm{ng} / \mathrm{mL})$ & $138.7 \pm 16.7$ & $136.1 \pm 12.7$ & 0.900 \\
Peak height $(\mathrm{ng} / \mathrm{mL})$ & $172.9 \pm 13.8$ & $175.9 \pm 14.2$ & 0.881 \\
Reactivity $(\mathrm{ng} / \mathrm{mL})$ & $34.2 \pm 10.3$ & $39.8 \pm 8.3$ & 0.673 \\
AUC $\left(\mathrm{ng}{ }^{*} \mathrm{~min} / \mathrm{mL}\right)$ & $-433.1 \pm 1045$ & $-263.3 \pm 822$ & 0.900 \\
\hline * Univariate analysis of variance & &
\end{tabular}

$614 \quad \mathrm{AUC}=$ Area under the curve 
616 Table 5: Mean $( \pm S E M)$ pre-treatment, peak height, reactivity and area under the

617 curve for heart rate and blood pressure in lower fitness and higher fitness women

\begin{tabular}{|c|c|c|c|c|}
\hline & & $\begin{array}{l}\text { Lower fitness } \\
(n=22)\end{array}$ & $\begin{array}{l}\text { Higher fitness } \\
(n=22)\end{array}$ & $p$ value* \\
\hline \multirow[t]{4}{*}{ SBP } & Pre-treatment $(\mathrm{mmHg})$ & $112 \pm 4$ & $104 \pm 2$ & 0.115 \\
\hline & Peak height $(\mathrm{mmHg})$ & $125 \pm 4$ & $116 \pm 3$ & 0.057 \\
\hline & Reactivity (mmHg) & $14 \pm 3$ & $12 \pm 3$ & 0.642 \\
\hline & $\mathrm{AUC}\left(\mathrm{mmHg}^{*} \mathrm{~min}\right)$ & $188 \pm 163$ & $104 \pm 118$ & 0.688 \\
\hline \multirow[t]{4}{*}{ DBP } & Pre-treatment $(\mathrm{mmHg})$ & $65 \pm 2$ & $62 \pm 2$ & 0.374 \\
\hline & Peak height $(\mathrm{mmHg})$ & $72 \pm 2$ & $72 \pm 3$ & 0.833 \\
\hline & Reactivity (mmHg) & $8 \pm 2$ & $10 \pm 3$ & 0.607 \\
\hline & $\mathrm{AUC}\left(\mathrm{mmHg}^{*} \min \right)$ & $-166 \pm 90$ & $-216 \pm 112$ & 0.730 \\
\hline \multirow[t]{4}{*}{ MAP } & Pre-treatment $(\mathrm{mmHg})$ & $82 \pm 3$ & $77 \pm 2$ & 0.119 \\
\hline & Peak height $(\mathrm{mmHg})$ & $90 \pm 3$ & $87 \pm 3$ & 0.507 \\
\hline & Reactivity (mmHg) & $7 \pm 2$ & $10 \pm 3$ & 0.503 \\
\hline & $\mathrm{AUC}\left(\mathrm{mmHg}^{*} \mathrm{~min}\right)$ & $-148 \pm 115$ & $-165 \pm 124$ & 0.920 \\
\hline \multirow[t]{4}{*}{ HR } & Pre-treatment (bpm) & $69 \pm 2$ & $64 \pm 2$ & 0.113 \\
\hline & Peak height (bpm) & $80 \pm 2$ & $71 \pm 2$ & 0.005 \\
\hline & Reactivity (bpm) & $11 \pm 2$ & $7 \pm 1$ & 0.084 \\
\hline & AUC (bpmmin) & $239 \pm 129$ & $54 \pm 69$ & 0.212 \\
\hline
\end{tabular}

*Univariate analysis of variance

619 SBP = systolic blood pressure, $\mathrm{DBP}=$ diastolic blood pressure, $\mathrm{MAP}=$ mean arterial

620 pressure, $\mathrm{HR}=$ heart rate 


\section{Figure captions}

623 Figure 1: Mean ( \pm SEM) plasma cortisol concentrations in lower and higher fitness

624 women from 1145h-1400h. The box labelled "lunch" represents the timing of the

625 lunch period and the hashed box represents the timing of the break to use the

626 bathroom

627

628 Figure 2: Mean ( \pm SEM) a) systolic, b) diastolic and c) mean arterial pressures and 629 d) heart rate in lower fitness and higher fitness women from 1145h-1400h. The 630 boxes labelled "lunch" represent the timing of the lunch period and the hashed boxes 631 represent the timing of the break to use the bathroom.

632 


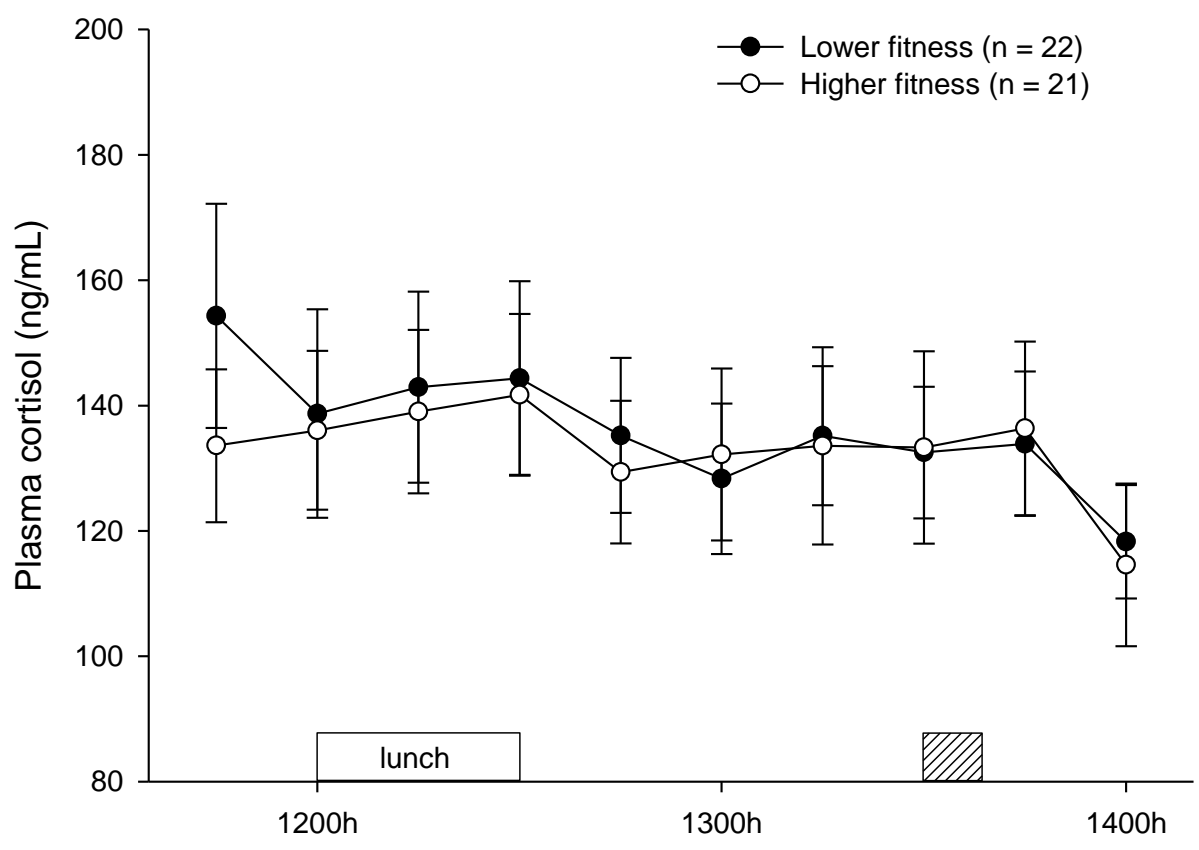




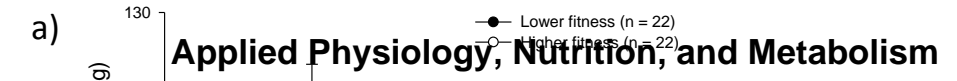

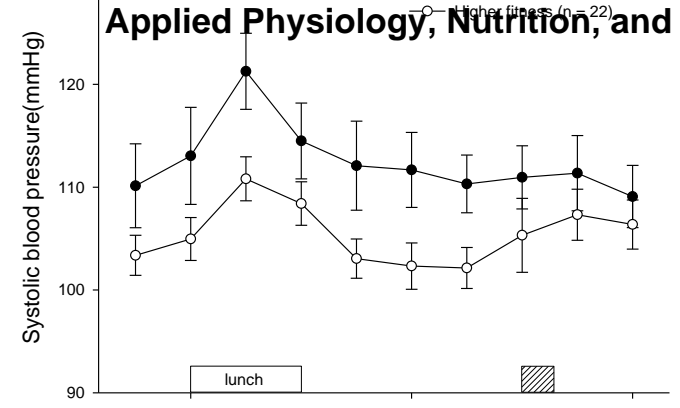

b)

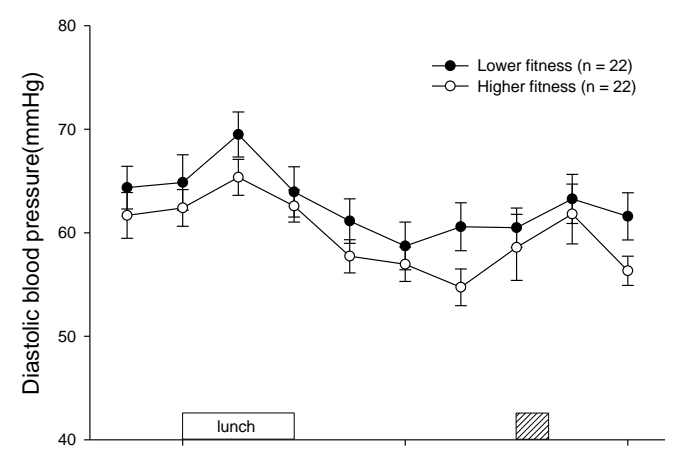

c)

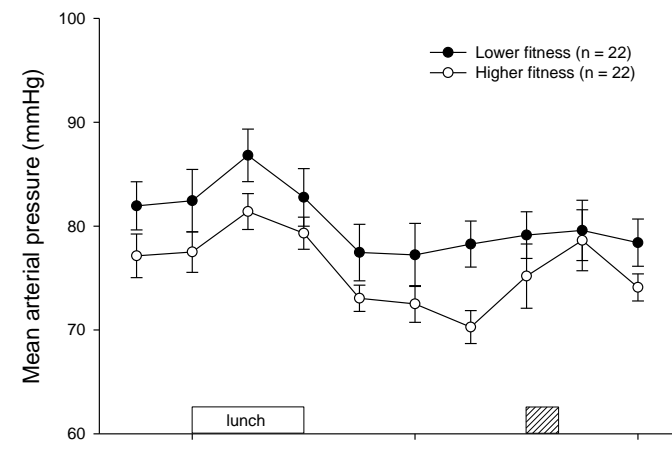

d)

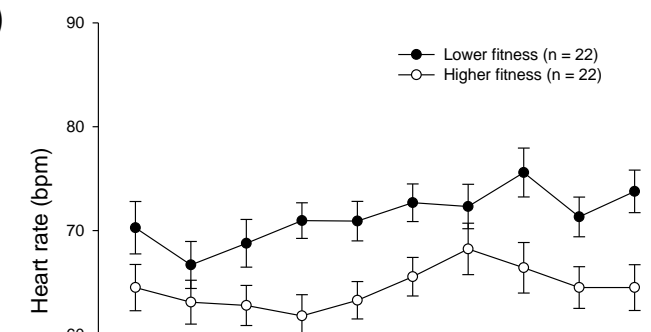

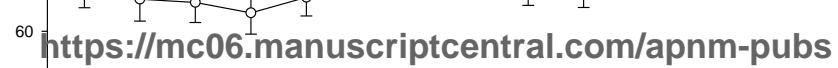

${ }_{50}$ 